\title{
'When Hunger Makes Everything Better Looking!: The Effect of Hunger on the Aesthetic Appreciation of Human Bodies, Faces and Objects
}

\section{Valentina Cazzato ( $\nabla$ v.cazzato@ljmu.ac.uk )}

Liverpool John Moores University

\section{Carmelo M. Vicario}

University of Messina

Cosimo Urgesi

IRCCS E. Medea

\section{Research Article}

Keywords: fasting, snack, body mass index, body appreciation, appetite

Posted Date: January 3rd, 2022

DOI: https://doi.org/10.21203/rs.3.rs-1151814/v1

License: (1) This work is licensed under a Creative Commons Attribution 4.0 International License.

Read Full License

Version of Record: A version of this preprint was published at BMC Psychology on April 11th, 2022. See the published version at https://doi.org/10.1186/s40359-022-00807-7. 


\section{Abstract}

Background: Research evidence suggests that physiological state of hunger might affect preference for female body weight, such that hungrier, compared to satiate, men prefer heavier body weight and rate as more attractive heavier female figures. Here, we seek to extend these findings by comparing the effects of fasting and snack on aesthetics judgements of the bodies and faces of conspecifics and of objects in a sample of female and male participants.

Methods: Forty-four participants (women: $\mathrm{n}=21$, mean age $=23.70 \mathrm{yrs} \pm 0.62$ ) provided aesthetic liking judgments of round and slim human bodies, faces and objects, under at least $12 \mathrm{~h}$ of overnight fasting and immediately after having eaten a snack (i.e., bananas). An anthropometric measure of adiposity (i.e., Body Mass Index, BMI) was also collected from each observer.

Results: Overall, we found that participants' aesthetic judgements were higher for slim stimuli compared to round ones. However, after fasting, participants rated round stimuli as more attractive compared to when they had a snack. This hunger-based shift in ratings not only was apparent when stimuli depicted a human body or face, but also when they depicted an object, thus suggesting a general modification of observers' aesthetic preference related to hunger. Importantly, this effect was modulated by participants' BMI so that only participants with a high BMI provided higher aesthetic judgements for round stimuli after fasting than after a snack.

Conclusions: Our results demonstrated that both the modification of the physiological state and the individual differences in adiposity level of the observers might affect the aesthetic appreciation of the external world.

\section{Introduction}

Hunger offers the strongest homeostatic motivation for behaviour in the animal kingdom, including humans. From an ethological perspective, animals must select and pursue food in times of caloric insufficiency, despite the wide diversity of stimuli and competing demands that naturally have a bearing on them. To address this homeostatic imbalance, animals must be able to successfully navigate their environment in ways that require them to flexibly switch between exploratory, defensive, and competing behaviours, indicating tremendous plasticity in feeding behaviour. Interestingly, a relevant study on mice supports this notion by reporting that, when mice are motivated to pursue other needs, such as water consumption, self-preservation in fear-inducing contexts, or social interaction with conspecifics, hunger overrides competing incentives to promote feeding behaviour [1].

Despite humans might more likely, as compared to other animals, to abstain from eating for a short period of time, for example for religious compliance or weight-loss dieting, even when access to food might not be that limited, hunger is nevertheless a primary need that takes priority in perception, action and cognition. It is known that calorie intake reduction and associated sensations of hunger have an impact on human cognition, including attention, memory, and executive function (see [2] for an extensive 
review). In particular, it has been widely reported that fasted individuals are more attracted to food [3] and more motivated to food acquisition and consumption [4] as compared to satiated ones. However, they are also more attracted to money [5], which can let them to acquire food, and have greater desire to possess various objects, to acquire non-food items, and also to take more samples of non-food objects [6]. Thus, hunger seems to lead to a general increase of acquisition-related attitudes and behaviours, influencing domains that are not relevant for reducing hunger [6]. In a similar vein, an observer's level of hunger may also influence the perception of others' actions when they are directed to not only a primary- (e.g., a muffin) or secondary-reinforced (i.e., a banknote) object, but also to a neutral object (e.g., a notepad; [7]). Finally, there is evidence that hunger can affect human decision-making in the field of morality, by reducing disapproval of ethical violations (e.g., $[8,9])$.

Interestingly, researchers have also reported the impact of hunger on other types of decisional processes such as the appreciation of human physical attractiveness, with most of them focussing on women's bodies and on men's ratings. For example, evidence to suggest a link between appetite and body image perception has been reported by a series of studies by Swami and Tovée [10] and Nelson and Morrison [11]. In their study, Nelson and Morrison [11] showed that men who were about to eat an evening meal rated as more attractive a heavier female body weight compared to men who had just eaten an evening meal, whereas women's rating of men's ideal body weight did not vary with those women's meal status. Swami and Tovée [10] asked hungry and satiated participants to rate a series of photographs of women with known body weight and shape. Corroborating the previous findings from Nelson and Morrison's study [11], their results indicated that before dining men preferred slightly heavier women (measured in terms of their Body Mass Index, BMI) than after dining. Notably, hungrier men also rated overweight and obese women more favourably. In keeping with these findings, a further study by Pettijohn, Sacco and Yerkes [12] asked men to select the weight category in pounds of an ideal female partner and found that hungry men gave more positive ratings to heavier, as compared to slimmer women.

All of these converging results (but see [13] for a non-replication in a randomized controlled betweensubject study) have been interpreted in light of the so called 'Insurance Hypothesis' [14]. According to this view, individuals who experience scarcity of resource, as when they feel hungry, respond to body adiposity as a protection from food scarcity. This shifts the aesthetic preference toward rounder body figures [10, $11,15,16]$. The relation between the experience of hunger and a more favourable perception of body adiposity, however, would predict that only the aesthetic judgment of human bodies is affected by hunger, while the perception of other objects should not. In keeping with this prediction, a series of studies by Swami et al. [15] asked hungry men to provide aesthetic judgments for a series of images of anvils or empty milk bottles of different sizes, or of milk bottles of different fill levels (empty to full) and did not find evidence to suggest that hunger influenced judgements of these other types of objects. Accordingly, Xu et al. [6] found that while hunger increased both the intention to acquire and the liking of food items, it promoted only the acquisition, but not the liking of non-food objects. However, Saxton et al. [16] have recently tested the effects of self-reported feeling of hunger on the attractiveness judgements of female and male bodies manipulated to vary in level of adiposity and of objects manipulated to vary in size. They found that, even if the effects of hunger were greater for bodies, larger sizes of stimuli in general 
were judged as more attractive by hungrier participants, thus questioning the body-selective effects of hunger on aesthetic preference.

Nevertheless, correlating individual sense of hunger in uncontrolled situations rather than manipulating food intake condition might confound hunger signals with interindividual variabilities in the experience and report of hunger. Indeed, people may differ in their experience of hunger for many reasons. For example, individual differences in the interoceptive states of hunger might concern the ability of detecting visceral signals and their changes, as well as the experience of differing motivational, affective and cognitive states under similar physiological input (see [17] for an extensive review on this topic). Personality traits such as cognitive restraint, which is the degree to which a person consciously regulates their food intake, may also mediate attention to interoceptive state of hunger $[18,19]$. With these regards, a previous study by Lattimore [20] suggested that adiposity, as measured by BMI, rather than dietary restraint might be an important mediator of appetite-specific body dissatisfaction and aesthetic preference. Accordingly, the study by Jin and colleagues [21] showed that it was not the starved or fed condition to which two groups of participants were randomly allocated, but their BMI that influenced their ratings of female attractiveness.

To further address the specificity of the effects of hunger on body weight perception, here, we devised a within-subject, lab-based study gathering aesthetic ratings of body and object stimuli in both male and female participants. Crucially, we also included another type of body-related stimuli, namely faces, which can serve as a proxy for the estimation of facial adiposity [22]. Particularly, we aimed to investigate whether physiological conditions of fasting and snack might affect participants' aesthetic ratings of liking when judging the appearance of thin and round bodies and faces, as compared to a familiar, control object (i.e., a vase). To address the limitations of between-subject $[10-13,15]$ or correlational [16] designs employed by the previous studies and to control for interindividual differences in the experience of hunger, the same participants provided aesthetic judgements of the human body under two different physiological conditions (in two different days): while being in food deprivation (i.e., after at least $12 \mathrm{~h}$ of fasting) and after having consumed a snack (i.e., after at least $12 \mathrm{~h}$ of fasting). Importantly, in each physiological condition, participants were asked to provide their subjective visual analogue scale (VAS) hunger ratings with the aim to disentangle the effect of food deprivation/intake from the effect due to changes in the hunger visceral sensation associated with the two physiological conditions $[7,8]$. Finally, we also controlled for participants' BMI that is known to affect hunger perception and physical attractiveness $[7,13]$.

Capitalising on previous literature, we expected our participants to provide higher liking judgments to slim than to round models [23-25]. However, we anticipated this effect to be influenced by participants' physiological state of hunger, so that participants will provide higher liking ratings to round stimuli when they are hungry as compared to when they are satiated. More specifically, in line with the predictions of the Insurance Hypothesis' [14] and previous related findings [10,11, 15], we expected that the effects of hunger should be specific for those stimuli that convey information about body adiposity, mainly bodies and, at least partially, faces, but not for object size, which is unrelated to body adiposity. Conversely, if 
hunger exerts a domain-general bias in bountifulness appreciation, shifting preferences toward bigger stimuli in general [16], then comparable effects of hunger should be obtained for all object categories. Finally, we also expected perceived hunger and individual BMI to moderate the effects of the physiological status of hunger with greater increase of aesthetic appreciation of round stimuli in hungrier $[11,16]$ and heavier $[7,20]$ participants.

\section{Methods}

\section{Participants}

The sample size for our $2 \times 3 \times 2$ ANCOVA design (numerator $d f=2 ; 3$ covariates) was based on a preliminary calculation using the freely available $G *$ Power software (G*Power 3.1.9; [26]), which indicated a minimum sample of 44 participants as adequate for a design with $85 \%$ power to detect a large effect size $(f=0.52)$, with alpha at 0.05 (two tailed). The effect size was estimated by averaging the effect size of the two studies reported in Nelson and Morrison ([11] study $1, \eta p^{2}=0.017$; study $2, \eta p^{2}=0.018$ ) and that reported by Swami and Tovee' ([10]; $\left.\eta p^{2}=0.6\right)$. A total of 44 participants (women: $\mathrm{n}=21$, mean age = $23.70 \mathrm{yrs} \pm 0.62 ;$ mean BMI $=23.25 \mathrm{~kg} / \mathrm{m}^{2} \pm 0.49$ ) from Liverpool John Moores University (LJMU) participated in the experiment in return for course credits or $£ 5$ shopping vouchers. All subjects but eight were right-handed as assessed by the Edinburgh Handedness Inventory [27]. Participants were asked to provide their self-identified gender identity (and not their biological sex), which was assessed through two forced-choice boxes (female, male). Participants (self)reported normal or corrected to normal vision and they were in good health, were free of psychotropic or vasoactive medication, with no current or history of psychiatric or neurological disease. Participants reporting specific diet requirements and/or allergies to plantain-based foods, being pregnant or diabetic (self)excluded from this investigation. All participants gave their written informed consent, and all were debriefed at the end of the experiment. All procedures were approved by the university research ethics committee of LJMU, in agreement with the ethical standards of the 1964 Declaration of Helsinki.

\section{Experimental stimuli}

Computer-generated imagery (CGI) was used to create 3D stimuli of bodies, faces and familiar objects (i.e., vases), which were designed by means of Poser Pro 2010 (e-frontier, Santa Cruz, CA) and were taken from the stimulus set of a previous study by our group (see [23] for specific details). 3D body stimuli consisted of two female and two male models, and they were wearing identical underwear black clothing. All models were standing against a grey background. Furthermore, each body model was displayed in different postures, from a frontal or three-quarter perspective. The apparent body weight of each model varied according to two levels of round and thin figures. In all models, the face was scrambled to rule out the impact of face identity discrimination in performing the body inversion task. 
Like the body stimuli, CGI model of 3D facial stimuli were selected from a set of six-dimensional adult face stimuli, taken from a previous study by our group [28]. The final set of stimuli consisted of two female and two male 3D faces. Like the body stimuli, faces were depicted against a grey background. Furthermore, we removed the hair to avoid any confound on perceived attractiveness. Each 3D model face displayed two different neutral expressions and was viewed from a frontal or three-quarter perspective. The level of adiposity of the faces was also manipulated to depict two levels of round and thin appearance.

Finally, for the object stimuli, we used virtual objects that depicted seven different virtual exemplars of vases. To match our object stimuli with the body and face stimuli perceptual features, each of these vases was presented from two different views and rendered against a grey background. Crucially, the size of each object exemplar was altered to reflect two levels of round and thin shape. Body, face and object stimuli were presented in three separate blocks. The order of the blocks was counterbalanced betweenparticipants, but it remained the same for the two hunger conditions of the same participant. Before the start of each experimental block, participants were introduced to the aesthetic task and presented with five practice trials (taken from a different set of body, face and object stimuli to avoid familiarisation), which were not considered in the main analyses. Each block was composed by two sub-blocks, each comprising 32 slim and 32 round stimuli, which were randomly presented (for a total of 128 stimuli per block). The participants could rest between blocks for how long they needed, usually no longer than $60 \mathrm{sec}$.

\section{General Procedure}

The experiment was performed by means of E-Prime software (version 2.0 Professional, Psychology Software Tools, Inc., Pittsburgh, PA) running on a Windows PC. During the experimental sessions, all participants were required to seat approximately $57 \mathrm{~cm}$ in front of a 15.6-inch LCD monitor (resolution, $1,024 \times 768$ pixels; refresh frequency, $60 \mathrm{~Hz}$ ), and were asked to complete an aesthetic (Liking) task. During each trial, participants were presented with a black central fixation cross on a grey background (see Fig. 1). After $500 \mathrm{~ms}$, a body (or face or object) appeared for $500 \mathrm{~ms}$ at the center of the screen subtending a visual angle of approximately $12^{\circ} \times 10^{\circ}$. Then, the stimulus was replaced by a random-dot mask obtained by scrambling the corresponding sample stimulus by means of custom-made image segmentation software (Matlab 9.5, The Mathworks, Inc., Natick, MA, USA) which lasted on the screen for $500 \mathrm{~ms}$. Finally, participants were prompted to provide their ratings by answering to the question 'How much do you like this body (or face or object)?' which also appeared at the center of the screen. The question was always presented along with a horizontal, $10 \mathrm{~cm}$ VAS ranging from 'very much' (100) to 'not at all' (0). The right- and left-side position of the anchor words of the VAS was balanced across participants.

Based on the successful experimental manipulation of hunger adopted by the study of Vicario and colleagues [7], we required participants to complete the aesthetic task in two different occasions, with 
each one corresponding to two different days. The two visits were always scheduled at morning time (i.e., between 08:00 am and 11:30 am) after at least a break of $24 \mathrm{~h}$ in between. During the two visits, participants were required to eat their last meal at least $12 \mathrm{~h}$ before the scheduled sessions. During a fasting session, participants completed the aesthetic task after at least $12 \mathrm{~h}$ of overnight fasting; during a snack session, participants completed the aesthetic task immediately after having eaten a snack (i.e., bananas). Participants were allocated to one or the other order of physiological status condition in a random manner. The order of fasting/snack sessions among participants was counterbalanced, so that half of participants started the first session after at least $12 \mathrm{~h}$ of sleep but before having eaten the snack (fasting session); and the second session after at least $12 \mathrm{~h}$ of sleep (snack session) but immediately after having eaten the snack. The other half of participants completed the task in the reversed order. Participants were invited to eat the snack until they felt satiated (for a maximum of two bananas). Following this, they were asked to provide hunger ratings both before and after breaking the fast using a horizontal 10-cm VAS, with anchor points labelled "Not at all hungry" to "Extremely hungry". Upon completion of the experimental tasks, anthropometric measure of adiposity, that is Body Mass Index $\left(\mathrm{BMl} ; \mathrm{kg} / \mathrm{m}^{2}\right)$ was obtained from measuring weight $(\mathrm{kg})$ and height $(\mathrm{cm})$, by means of a scale and a stadiometer. Overall, testing lasted approximatively $45 \mathrm{~min}$.

\section{Data handling}

All statistical analyses were performed using STATISTICA 8.0 (StatSoftInc, Tulsa, Oklahoma). First, a series of two-tailed t-tests were performed to compare appetite ratings in the fasting and the snack conditions. Second, the mean Liking VAS scores were entered in a 2 (fasting, snack - physiological status) $\times 3$ (body, face, object - stimuli) $\times 2$ (round, slim - size) repeated measures analyses of covariance (ANCOVA), entering participants' BMI, appetite ratings in the fasting and the snack conditions as continuous predictors. This way, we could test not only the effects of food depletion and intake (i.e., in association to fasting vs. snack condition), but also those of the individual perceived level of hunger in each condition. Significant interactive effects of a covariate with the within-subject variables were explored by assessing the effects of the within-subjects variables (or their interaction) on the dependent variable at particular levels of the covariate using a mixed-model ANOVA design (Green \& Salkind, 2011), by splitting the participant sample into two groups based on the median of the covariate distribution. All data are reported as Mean (M) and Standard Error of the Mean (S.E.M.). A significance threshold of $p<$ 0.05 was set for all effects and effect sizes were estimated using the partial eta square measure $\left(n p^{2}\right)$. Duncan post-hoc tests were performed to follow-up significant interactions.

\section{Results}

Manipulation of hunger and satiety worked as expected. Indeed, the two tailed $t$ tests showed significant higher self-reported hunger ratings in the fasting condition $(60.15 \pm 25.03)$, compared to the snack (39.94 $\pm 29.48)$ condition $\left[t_{(43)}=4.35, p<0.001\right]$. 


\section{Aesthetic Task}

The ANCOVA on the liking ratings revealed a main effect of size, which indicated that, overall, slim stimuli were liked more that round stimuli $\left[F_{(1,40)}=11.014, p=0.002, \eta p^{2}=0.216\right]$. The main effect of type of stimuli was moderated by the covariate of hunger ratings for the snack condition $\left[F_{(2,80)}=4.270, p=\right.$ $\left.0.017, n p^{2}=0.096\right]$. A two-way interaction of type of stimuli by size was observed $\left[F_{(2,80)}=5.178, p=\right.$ $0.008, \eta p^{2}=0.115$, see Fig. 1]. Post hoc analyses revealed that round stimuli were always liked less than slim stimuli (all $p s<0.001$ ). However, round objects were liked significantly more compared to round bodies and faces (all $p s<0.001$ ), whose appreciation in turn did not differ $(p=0.771)$. Conversely, slim bodies were liked significantly more than slim faces and objects (all $p s<0.001$ ), whilst no significant difference in the liking ratings was observed between slim faces and objects $(p=0.287)$.

Notably, the significant main effect of size was also moderated by the covariate of participants' BMI $\left[F_{(1,40)}=4.127, p=0.049, \eta p^{2}=0.094\right]$, which importantly was further qualified by a significant

interaction of physiological status, size and participants' BMI $\left[F_{(1,40)}=6.053, p=0.018, \eta p^{2}=0.131\right]$. This suggested that the experimental manipulation of the physiological status had different effects on participants' liking ratings of round and slim stimuli depending on participants' BMIs.

To explore the source of the significant modulation of participants' BMI on the interaction between physiological status $\times$ size, we split participants into two groups (Low BMI [n $=22]$ \& High BMI [n = 22]) based on the median BMI score (med $=22.63$, Low BMI: mean $=20.48 \mathrm{~kg} / \mathrm{m}^{2} \pm 0.28$ \& High BMI: mean $=$ $26.02 \mathrm{~kg} / \mathrm{m}^{2} \pm 0.43$, respectively). Therefore, we conducted two separate follow-up mixed model ANOVAs with physiological status and size as within subject variables, respectively for High vs. Low BMI participants' groups. The ANOVAs revealed a significant interaction between the two within-subject variables only for the high $\mathrm{BMI}$ group $\left[F_{(1,20)}=10.647, p=0.004, \eta p^{2}=0.347\right]$, Importantly, post hoc analysis showed that participants with a high BMI liked more slim than round stimuli during both fasting and snack conditions (all $p s>0.001$ ). On the other hand, round stimuli were liked more in the fasting $(37.40 \pm 1.63)$ than in the snack condition $(35.07 \pm 1.97 ; p=0.001)$. No difference was observed between the liking ratings of slim stimuli in the fasting and snack conditions $(50.94 \pm 1.53$ vs. $51.07 \pm 1.92, p=$ 0.683). For the low BMI group, a significant main effect of size was observed $\left[F_{(1,22)}=87.181, p<0.001\right.$, $\left.\eta p^{2}=0.798\right]$. However, neither the main effect of physiological status $\left[F_{(1,22)}=0.325, p=0.574, \eta p^{2}=\right.$ $0.014]$ nor the interaction of physiological status by size was were significant $\left[F_{(1,22)}=0.050, p=0.825\right.$, $\eta p^{2}=0.002$, see Fig. 2] in the low BMI group.

\section{Discussion}

In the current research, we aimed to extend and qualify previous evidence of an effect of physiological states of hunger on the aesthetic appreciation of human bodies. In particular, we tested the specificity of the increased appreciation of roundness under starvation for those stimuli that convey information about 
body adiposity, namely bodies and, indirectly, faces, but not for other objects, such as vases, whose size is unrelated to fat storage. To this aim, we used a within-subject design, in which the same participants rated how much they liked slim and round bodies, faces and vases after a period of $12 \mathrm{~h}$ fasting or after having interrupted a comparably long fasting with a (controlled) snack. Furthermore, we also controlled for individual differences in perceived hunger and BMI. Our results replicated the findings that aesthetic appreciation of human bodies can be indeed influenced by physiological states of hunger. Importantly, we added to the current picture by showing that not only bodies, but also faces, which may provide an at least indirect clue to fat storage, and objects, whose size is unrelated to fat storage, might be subjected to hunger-induced preference shifts towards roundness. Crucially, we also showed that these shifts for rounder stimuli can be influenced by individual differences in anthropometric measures of adiposity, as measured with BMI. With these regards, we found that despite all participants showing stronger liking preferences for slimmer stimuli, only individuals with high BMI exhibited a hunger-induced preference shift towards roundness. No such a shift was observed in the low BMI group.

Several previous studies have shown that rounder bodies are rated as more attractive and liked more by hungry than satiated observers $[10,11,15,16]$. This evidence has been interpreted within the Insurance Hypothesis [14], according to which individuals should respond to food insecurity cues, as those conveyed by hunger, by experiencing psychological and behavioural changes that promote increased fat storage. Such changes are supposed to be driven by the increased survival afforded by fat stores in buffering against energy shortfalls during times of food scarcity. Accordingly, it might be plausible that to counter possible future shortages in food supply, individuals must adapt by shifting their preference for rounder bodies under conditions of hunger. Thus, hunger-linked shifts in judgements of ideal body size are thought to favour this adaptation $[10,11,15]$. This view has been also called into action to explain cultural differences in ideal body weight [14].

Importantly, whilst the Insurance Hypothesis does not make specific claims about the specificity of the effects of food scarcity on hunger-induced shifts towards rounder faces, it can accommodate our finding that, when fasting, high-BMI participants preferred rounder faces after fasting than after a snack. This does not come at surprise considering that face adiposity can influence person attractiveness as a proxy of body adiposity [22]. Indeed, several lines of research suggested that faces and bodies provide somewhat overlapping but also uniquely valuable pieces of socially relevant information. For example, studies have also shown that women's body size, as indexed by BMI, provides significant cues of their current fertility, pregnancy status, and ability to support foetal development [29-33]. In a similar vein, regarding facial cues, women's faces provide significant cues of their health, age, femininity, and personality traits [34-37]. Previous studies also supported the notion that both body and face equally influence judgments of women's overall attractiveness [38, 39]. With these regards, a study by BleskeRechek et al. [40] reported that ratings of women's bodily and facial attractiveness independently predicted the overall ratings of person attractiveness, thus suggesting that attributes of face and body may share some underlying factor of genetic quality that is perceived as attractive [41]. Accordingly, increased preference for rounder faces and bodies in hungrier participants might be due to the genuine 
(and similar) importance of bodies and faces in conveying socially relevant information which are pivotal to human physical attractiveness and human mate choice decisions.

What can more loosely fit with the Insurance Hypothesis is the finding that food depletion also had an influence on objects, and not only on bodies and faces. Indeed, participants liked more round vases under starvation than after being satiated, and the effect for objects was comparable to that for bodies and faces. This agrees with and extend to a within-subject experimental design the findings of the correlational study by Saxon and colleagues [16], which found that hungrier people were more likely to select bigger objects, and not only rounder bodies, as more attractive. Nevertheless, it is worth noticing that this study still found greater effects of perceived hunger for body stimuli, especially female bodies, than for objects. This was partially in keeping with the results of Swami et al. [15], which failed to find evidence to suggest that hunger influences judgements of other types of objects. However, this null result was not contrasted, within the same study, with a significant effect of hunger on body appreciation, thus leaving the possibility of power issues. Here, using an experimental manipulation of hunger and a similar aesthetic (liking) judgement as in the study by Swami et al. ([15], how aesthetically pleasing objects are), we found comparable hunger effects on the appreciation of both round bodies (and faces) and round objects. This keeps with a general effect of hunger to domains that are irrelevant for food intake or fat storage, as suggested by the findings that hunger gives rise to a greater desire to possess and acquire more samples of non-food objects [6]. Thus, in accordance with Saxton et al. [16], it might be that hunger motivates people towards abundance in general, explaining why our participants liked more round stimuli in general, and not only round bodies and faces, when starving than when satiated. This does not necessarily contrast with the Insurance Hypothesis, but it may qualify it. Indeed, the effects of hunger may be routed in an increased sensitivity to cues of food scarcity, but they may extend well beyond food intake and body adiposity, generally driving sensitivity to bountifulness.

In this sense, the Insurance Hypothesis might help with explaining why hunger-induced preference shifts towards roundness of all stimuli were observed in individuals with higher BMI only. Indeed, it predicts that optimal level of body fat to store depends upon security of access to food and that higher body weights should be more common when food insecurity is high because that would make body mass safe. Individuals with higher BMI might perceive greater food shortfall and, thus, stronger shift towards a preference for rounder stimuli. However, the claim that food insecurity is a predictor of high body weights in humans is far from being conclusive. Indeed, the overall association may be moderated by differences in individuals' socio-economic status, sex and self-body perception [20,14], pointing to a multifactorial mechanism.

One alternative but not mutually explanation for the moderating role of BMI could be that it reflects a decreased quantity of food that was consumed in individuals with low vs. high BMI. This confound might indeed affect the quasi-experimental manipulation of testing participants before vs. after a self-served meal $[11,15]$. However, we believe this is not the case in our study, given that the stringent design adopted by our work ensured that participants ate the maximum amount of food in a laboratory setting. Accordingly, during our experimental manipulation of snack, all participants were offered the same type 
and quantity of food (i.e., maximum of two bananas), which is also considered a healthy (low calories) type of food, thus allowing for a more rigorous test of factors that might impact upon people's affective state and aesthetic appreciation.

There are some limitations to this work which warrant consideration. First, addressing the effects of gender (either of the observer or of the model) on hunger-induced shifts of aesthetic preference went beyond the scope of this investigation (and the number of participants per each gender group did not allow to test for this hypothesis). Thus, we cannot rule out that our results might be moderated by specific cognitive and neural organization of aesthetic body appreciation in male and female observers [25] or by the specific gender-typing features of the model body under evaluation [42]. Accordingly, previous works, which focussed predominantly on men's judgements of women's bodies, reported that the effect of hunger on judgements of women's body fatness was more dramatic than its effect on judgements of other stimuli [16]. Although in the current study, we took advantage of the use of computer-generated images to create alterable 3-D human figure models with the standard "emaciated" and "heavy" settings supplied by the software Poser Pro 2010 (e-frontier, Santa Cruz, CA, USA), which simulate fat distribution in a realistic way as adiposity increases (see [23] for details on the creation of stimuli), at present we do not know which specific bodily and face cues of our virtual models were particularly salient to participants when they were making their aesthetic ratings. To address these issues, future work might seek to uncover whether there are specific (perhaps sexual) cues of the body or face of conspecifics that go beyond size and body shape (e.g., muscularity) that are particularly relevant to aesthetic judgements depending on one's physiological hunger and the gender of the observers.

Nevertheless, the general shift toward roundness of the aesthetic preference of both human and object stimuli calls into play domain-general mechanisms for the effects of hunger on aesthetic appreciation.

One other limitation of our study is that we did not directly assess participants' socio-economic status or their financial satisfaction/security similarly to what has been done in previous work on this topic [11, 43]. Accordingly, the study by Nelson and Morrison [11] reported that either financially dissatisfied or hungry men preferred a heavier mate than did financially satisfied men or satiated men, respectively. However, the study by Swami, Tovée and Furnham [43] failed to replicate Nelson and Morrison [11]'s findings showing that financially dissatisfied men did not rate a heavier female body weight as more attractive than did financially satisfied men. In light of these contrasting findings, we cannot exclude that other sociodemographic variables, including participants' socio-economic status, might have affected the results of our study and future investigations are needed to better elucidate the role of financial security in shaping the aesthetic appreciation of the body depending on hunger sensation.

\section{Conclusions}

We provide evidence of general effects of food depletion/intake on the aesthetic judgements of faces and objects, which go far beyond the domain of body perception. We also show that participants with high BMI might be particularly susceptible to hunger-induced preference shifts of preference for body fatness, with greater appreciation of rounder stimuli in hungrier and heavier participants. On the contrary, 
participants with lower levels of body adiposity (low BMI) appeared to be more resistant to changes of their aesthetic ratings for rounder stimuli, regardless of their physiological states of hunger and satiation. Future work might seek to elucidate the relationship between physiological states of hunger and shifts in appreciation of the human bodies and whether this relationship might be mediated by individual traits associated to the beholder's body adiposity.

\section{Declarations}

\section{Ethics Approval and Consent to participate:}

Informed consent was obtained by all participants through written consent. The procedures were approved by the University Research Ethics Committee (UREC, approval n. 18/NSP/056) of Liverpool John Moores University (LJMU) and complied with the ethical standards of the 1964 Declaration of Helsinki.

\section{Consent for publication:}

Identifiable demographic information has been removed from this manuscript to ensure anonymity. Thus, the consent to publish is not applicable.

\section{Data availability:}

The datasets generated during and/or analysed during the current study are available in the form of supplemental materials.

\section{Competing interests:}

The authors declare that they have no competing interests.

\section{Funding:}

This research did not receive any specific grant from funding agencies in the public, commercial, or notfor-profit sectors.

\section{Authors' contributions:}

All authors conceived the study. VC performed data collection. VC and CU performed the analyses. The first draft of the manuscript was written by VC, with input from all authors. All authors approved the manuscript before submission. 


\section{Acknowledgments:}

The authors thank Muna Farah, Ashleigh Bellard and Alberto Failla for helping with data collection.

\section{References}

1. Burnett CJ, Li C, Webber E, Tsaousidou E, Xue SY, Brüning JC, Krashes MJ. Hunger-driven motivational state competition. Neuron. 2016 Oct 5;92(1):187-201.

2. Benau EM, Orloff NC, Janke EA, Serpell L, Timko CA. A systematic review of the effects of experimental fasting on cognition. Appetite. 2014 Jun 1;77:52-61.

3. Lozano DI, Crites SL, Aikman SN. Changes in food attitudes as a function of hunger. Appetite. 1999 Apr 1;32(2):207-18.

4. Nisbett RE, Kanouse DE. Obesity, food deprivation, and supermarket shopping behavior. Journal of Personality and Social Psychology. 1969 Aug;12(4):289.

5. Briers B, Pandelaere M, Dewitte S, Warlop L. Hungry for money: The desire for caloric resources increases the desire for financial resources and vice versa. Psychological science. 2006 Nov;17(11):939-43.

6. Xu AJ, Schwarz N, Wyer RS. Hunger promotes acquisition of nonfood objects. Proceedings of the National Academy of Sciences. 2015 Mar 3;112(9):2688-92.

7. Vicario $\mathrm{CM}$, Kuran $\mathrm{KA}$, Urgesi C. Does hunger sharpen senses? A psychophysics investigation on the effects of appetite in the timing of reinforcement-oriented actions. Psychological research. 2019 Apr;83(3):395-405.

8. Vicario CM, Kuran KA, Rogers R, Rafal RD. The effect of hunger and satiety in the judgment of ethical violations. Brain and cognition. 2018 Aug 1;125:32-6.

9. Kerry N, Loria RN, Murray DR. Gluttons for punishment? Experimentally induced hunger unexpectedly reduces harshness of suggested punishments. Adaptive Human Behavior and Physiology. 2019 Dec;5(4):352-70.

10. Swami V, Tovée MJ. Does hunger influence judgments of female physical attractiveness?. British Journal of Psychology. 2006 Aug;97(3):353-63.

11. Nelson LD, Morrison EL. The symptoms of resource scarcity: Judgments of food and finances influence preferences for potential partners. Psychological science. 2005 Feb;16(2):167-73.

12. Pettijohn II TF, Sacco Jr DF, Yerkes MJ. Hungry people prefer more mature mates: A field test of the environmental security hypothesis. Journal of Social, Evolutionary, and Cultural Psychology. 2009 Sep;3(3):216.

13. Jin Z, Wang G, Hu S, Speakman JR. No impact of hunger on male perception of female physical attractiveness in relation to adiposity: a randomized controlled trial. International Journal of Obesity. 2020 Feb;44(2):418-27. 
14. Nettle D, Andrews C, Bateson M. Food insecurity as a driver of obesity in humans: The insurance hypothesis. Behavioral and Brain Sciences. 2017;40.

15. Swami V, Poulogianni K, Furnham A. The influence of resource availability on preferences for human body weight and non-human objects. Journal of Articles in Support of the Null Hypothesis. 2006;4(1):17-28.

16. Saxton TK, McCarty K, Caizley J, McCarrick D, Pollet TV. Hungry people prefer larger bodies and objects: The importance of testing boundary effects. British Journal of Psychology. 2020 Aug;111(3):492-507.

17. Stevenson RJ, Mahmut M, Rooney K. Individual differences in the interoceptive states of hunger, fullness and thirst. Appetite. 2015 Dec 1;95:44-57.

18. Ogden J, Wardle J. Cognitive restraint and sensitivity to cues for hunger and satiety. Physiology \& behavior. 1990 Mar 1;47(3):477-81.

19. Carper JL, Fisher JO, Birch LL. Young girls' emerging dietary restraint and disinhibition are related to parental control in child feeding. Appetite. 2000 Oct 1;35(2):121-9.

20. Lattimore P. Body dissatisfaction in females varies according to deprivation state and is modified by weight rather than restraint status. Appetite (Print). 2005;45(3):356-9.

21. Jin Z, Wang G, Hu S, Speakman JR. No impact of hunger on male perception of female physical attractiveness in relation to adiposity: a randomized controlled trial. International Journal of Obesity. 2020 Feb;44(2):418-27.

22. De Jager S, Coetzee N, Coetzee V. Facial adiposity, attractiveness, and health: A review. Frontiers in psychology. 2018 Dec 21;9:2562.

23. Cazzato V, Siega S, Urgesi C. "What women like”: influence of motion and form on esthetic body perception. Frontiers in psychology. 2012 Jul 9;3:235.

24. Mele S, Cazzato V, Urgesi C. The importance of perceptual experience in the esthetic appreciation of the body. PLoS One. 2013 Dec 4;8(12):e81378.

25. Cazzato V, Mele S, Urgesi C. Gender differences in the neural underpinning of perceiving and appreciating the beauty of the body. Behavioural brain research. 2014 May 1;264:188-96.

26. Faul F, Erdfelder E, Lang AG, Buchner A. G* Power 3: A flexible statistical power analysis program for the social, behavioral, and biomedical sciences. Behavior research methods. 2007 May;39(2):17591.

27. Oldfield RC. The assessment and analysis of handedness: the Edinburgh inventory. Neuropsychologia. 1971 Mar 1;9(1):97-113.

28. Cazzato V, Walters ER, Urgesi C. Associations of observer's gender, Body Mass Index and internalization of societal beauty ideals to visual body processing. Psychological Research. 2021 Jan 12:1-4.

29. Jasieńska G, Ziomkiewicz A, Ellison PT, Lipson SF, Thune I. Large breasts and narrow waists indicate high reproductive potential in women. Proceedings of the Royal Society of London. Series B: 
Biological Sciences. 2004 Jun 22;271(1545):1213-7.

30. Lassek WD, Gaulin SJ. Waist-hip ratio and cognitive ability: is gluteofemoral fat a privileged store of neurodevelopmental resources?. Evolution and Human Behavior. 2008 Jan 1;29(1):26-34.

31. Singh D. Body shape and women's attractiveness. Human nature. 1993 Sep 1;4(3):297-321.

32. Tovée MJ, Reinhardt S, Emery JL, Cornelissen PL. Optimum body-mass index and maximum sexual attractiveness. The Lancet. 1998 Aug 15;352(9127):548.

33. Zaadstra BM, Seidell JC, Van Noord P, te Velde ER, Habbema JD, Vrieswijk B, Karbaat J. Fat and female fecundity: prospective study of effect of body fat distribution on conception rates. British medical journal. 1993 Feb 20;306(6876):484-7.

34. Boothroyd LG, Jones BC, Burt DM, DeBruine LM, Perrett DI. Facial correlates of sociosexuality. Evolution and Human Behavior. 2008 May 1;29(3):211-8.

35. Gray AW, Boothroyd LG. Female facial appearance and health. Evolutionary Psychology. 2012 Jan 1;10(1):147470491201000108.

36. Gangestad SW, Scheyd GJ. The evolution of human physical attractiveness. Annu. Rev. Anthropol.. 2005 Oct 21;34:523-48.

37. Mirams L, Poliakoff E, Zandstra EH, Hoeksma M, Thomas A, El-Deredy W. Feeling bad and looking worse: negative affect is associated with reduced perceptions of face-healthiness. PloS one. 2014 Sep 26;9(9):e107912.

38. Currie TE, Little AC. The relative importance of the face and body in judgments of human physical attractiveness. Evolution and human behavior. 2009 Nov 1;30(6):409-16.

39. Peters M, Rhodes G, Simmons LW. Contributions of the face and body to overall attractiveness. Animal Behaviour. 2007 Jun 1;73(6):937-42.

40. Bleske-Rechek A, Kolb CM, Stern AS, Quigley K, Nelson LA. Face and body: independent predictors of women's attractiveness. Archives of sexual behavior. 2014 Oct;43(7):1355-65.

41. Thornhill R, Grammer K. The body and face of woman: One ornament that signals quality?. Evolution and Human Behavior. 1999 Mar 1;20(2):105-20.

42. D'Argenio G, Finisguerra A, Urgesi C. Motion and Gender-Typing Features Interact in the Perception of Human Bodies. Frontiers in neuroscience. 2020 Apr 21;14:277.

43. Swami V, Tovée MJ, Furnham A. Does financial security influence judgements of female physical attractiveness?. The Journal of Socio-Economics. 2008 Aug 1;37(4):1363-70.

\section{Figures}




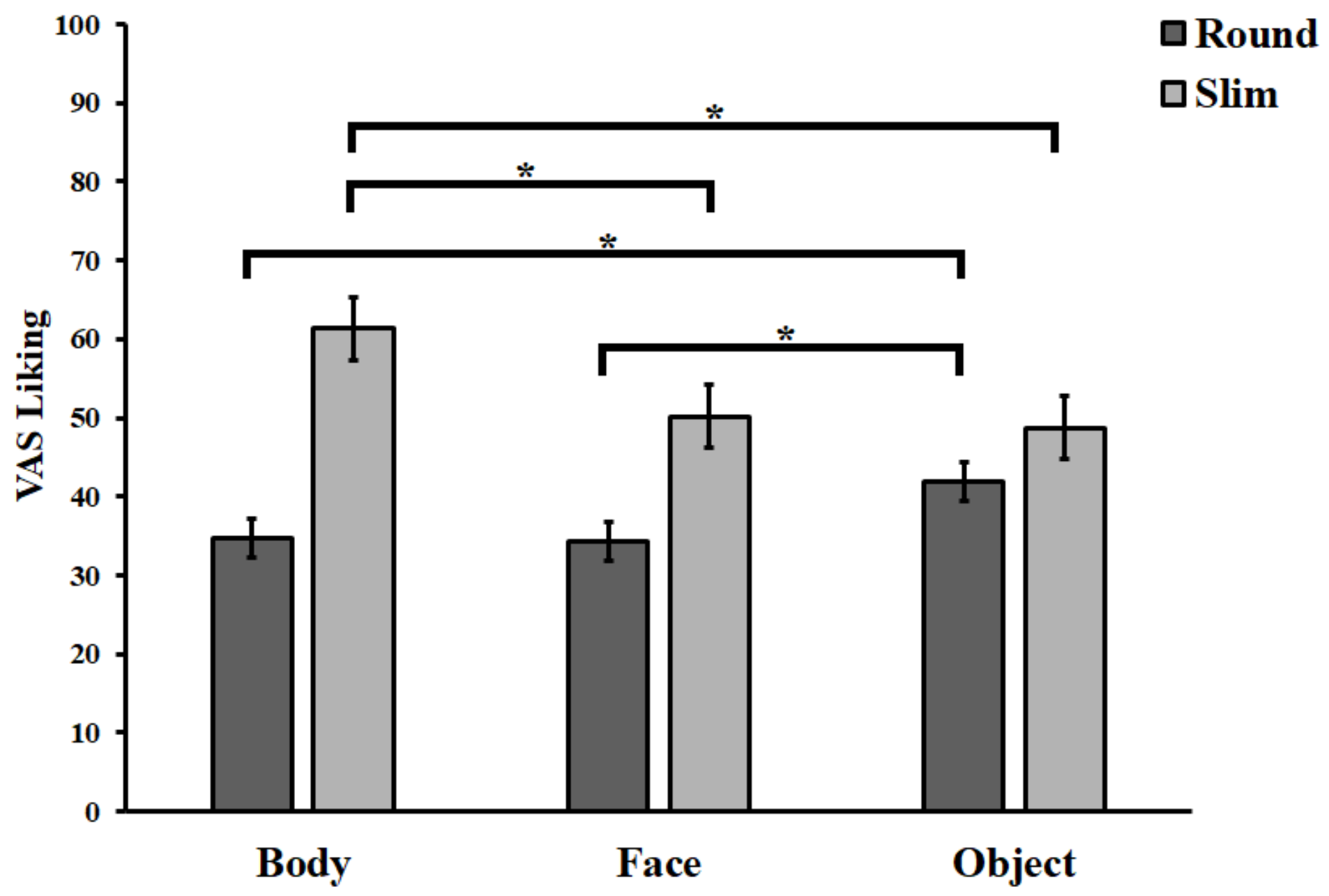

Figure 1

Mean ( \pm SEM) scores of the visual analogue scale (VAS) of the aesthetic judgements for the three types of stimuli (bodies, faces and object) for the two levels of roundness (round, slim). The asterisk symbol indicates a significant difference $\left({ }^{\star} p<0.05\right)$. 


\section{Low BMI Group}

Body

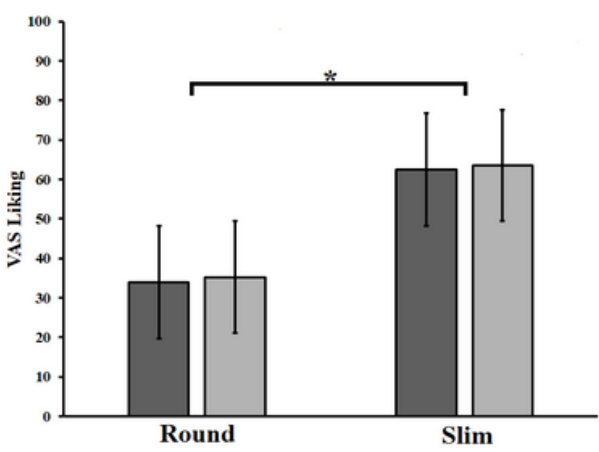

Body

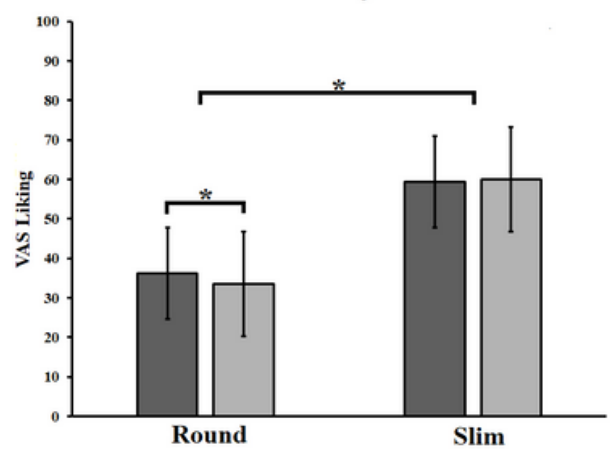

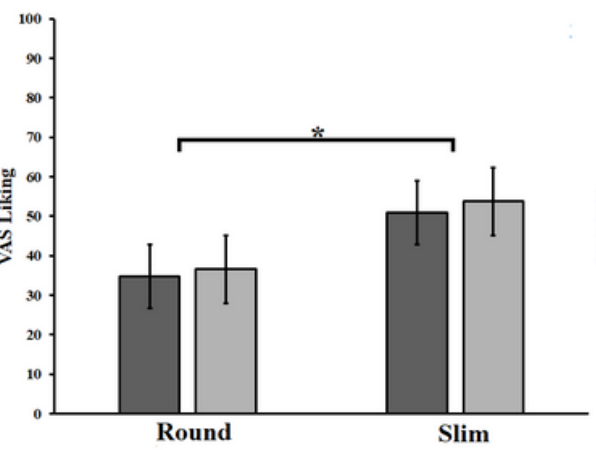

High BMI Group

Face

Face

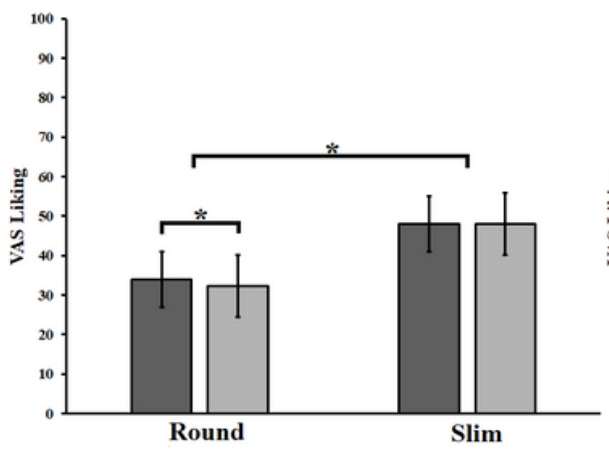

Object

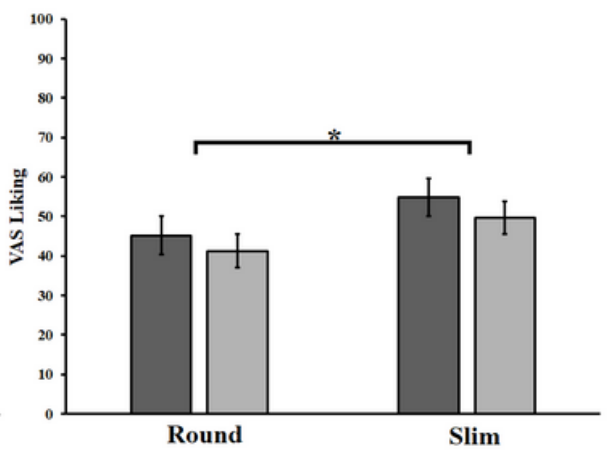

Object

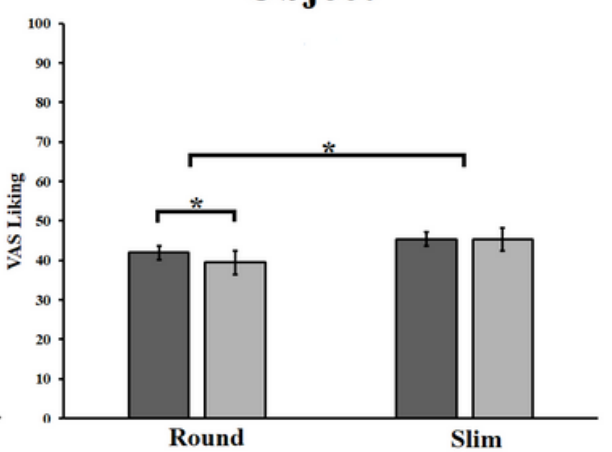

Figure 2

Mean ( \pm SEM) scores of the visual analogue scale (VAS) of the aesthetic judgements for the three types of stimuli (body, face and object), the two levels of roundness (round, slim) in the two physiological conditions (fasting, snack), for participants with low and high BMI. The asterisk symbol indicates a significant difference $\left({ }^{\star} p<0.05\right)$.

\section{Supplementary Files}

This is a list of supplementary files associated with this preprint. Click to download.

- DataHungerStudyf.xlsx 